\title{
On the lightness of chordal 4-cycle in 1-planar graphs with high minimum degree*
}

\author{
Xin Zhang ${ }^{\dagger}$ \\ Department of Mathematics, Xidian University, Xi'an 710071, P. R. China \\ Guizhen Liu $\ddagger$ \\ School of Mathematics, Shandong Univeristy, Jinan 250100, P. R. China
}

Received 12 January 2012, accepted 8 February 2013, published online 7 May 2013

\begin{abstract}
A graph $G$ is 1-planar if it can be drawn on the plane so that each edge is crossed by at most one other edge. The family of 1-planar graphs with minimum vertex degree at least $\delta$ and minimum edge degree at least $\varepsilon$ is denoted by $\mathcal{P}_{\delta}^{1}(\varepsilon)$. In this paper, it is proved that every graph in $\mathcal{P}_{7}^{1}(14)$ (resp. $\mathcal{P}_{6}^{1}(13)$ ) contains a copy of chordal 4-cycle with all vertices of degree at most 10 (resp. 12).
\end{abstract}

Keywords: 1-planar graph, light graph, cycle, discharging.

Math. Subj. Class.: 05C75, 05C10

\section{Introduction}

All graphs considered in this paper are finite, undirected, loopless and without multiple edges. For a graph $G$, we use $V(G), E(G), \delta(G)$ and $\Delta(G)$ to denote the vertex set, the edge set, the minimum degree and the maximum degree of $G$, respectively. By $F(G)$, we denote the face set of $G$ when $G$ is a plane graph. If $u v \in E(G)$, then $u$ is said to be the neighbor of $v$. We use $N_{G}(v)$ to denote the set of neighbors of a vertex $v$. The degree of a vertex $v \in V(G)$, denoted by $d_{G}(v)$, is the value of $\left|N_{G}(v)\right|$, and the degree of an edge $u v \in E(G)$, denoted by $d_{G}(u v)$, is the value of $d_{G}(u)+d_{G}(v)$. A $k-, k^{+}$- and $k^{-}$-vertex is a vertex of degree $k$, at least $k$ and at most $k$, respectively. In this paper, $C_{k}$ and $P_{k}$ denotes a cycle and a path with $k$ vertices and $K_{4}^{-}$denotes a chordal 4-cycle, which is a

\footnotetext{
* A project funded by Scientific Research Program of the Higher Education Institution of XinJiang (Grant No. XJEDU2012I38) and the Fundamental Research Funds for the Central Universities (Grant No. K5051370003).

${ }^{\dagger}$ Supported in part by the National Natural Science Foundation of China (Grants No. 11101243, 11201440).

${ }^{\ddagger}$ Supported in part by the National Natural Science Foundation of China (Grants No. 61070230).

E-mail addresses: xzhang@xidian.edu.cn (Xin Zhang), gzliu@sdu.edu.cn (Guizhen Liu)
} 
graph obtained by removing an edge from a complete graph $K_{4}$. A cycle $C=\left[x_{1} \cdots x_{k}\right]$ of a graph $G$ is of the type $\left(d_{1}, \cdots, d_{k}\right)$ if $d_{G}\left(x_{i}\right)=d_{i}$ for $1 \leq i \leq k$. Similarly we can define cycles of the type $\left(\geq d_{1}, \cdots, \geq d_{k}\right)$, etc. For other undefined concepts we refer the readers to [2].

A graph is 1-planar if it can be drawn on the plane so that each edge is crossed by at most one other edge. The notion of 1-planarity was introduced by Ringel [16] while trying to simultaneously color the vertices and faces of a plane graph $G$ such that any pair of adjacent/incident elements receive different colors. Note that we can construct, for given plane graph, a 1-planar graph $G^{1}$ whose vertex set is $V(G) \cup F(G)$ and any two vertices of $G^{1}$ are adjacent if and only if their corresponding elements in $G$ are adjacent or incident.

Borodin proved that each 1-planar graph is 6-colorable (the bound 6 being sharp) [3, 4] which positively answered a conjecture raised by Ringel in [16], and that each 1-planar graph is acyclically 20-colorable [7]. The list analogue of vertex coloring of 1-planar graphs was investigated by Albertson and Mohar [1], and by Wang and Lih [18]. Zhang et al. showed that each 1-planar graph $G$ with maximum degree $\Delta$ is $\Delta$-edge-colorable if $\Delta \geq 10$ [25], or $\Delta \geq 9$ and $G$ contains no chordal 5-cycles [19], or $\Delta \geq 8$ and $G$ contains no chordal 4-cycles [20], or $\Delta \geq 7$ and $G$ contains no 3-cycles [21], is $(\Delta+1)$ edge-choosable and $(\Delta+2)$-total-choosable if $\Delta \geq 16$ [27], is $\Delta$-edge-choosable and $(\Delta+1)$-total-choosable if $\Delta \geq 21$ [27]. Zhang et al. also showed that the $(p, 1)$-total labelling number of each 1-planar graph $G$ is at most $\Delta(G)+2 p-2$ if $\Delta(G) \geq 8 p+4$ [28], and the linear arboricity of each 1-planar graph $G$ is exactly $\lceil\Delta(G) / 2\rceil$ if $\Delta(G) \geq 33$ [24].

Another topic concerning 1-planar graphs is to investigate their global and local structures. In [9], it is shown that each 1-planar graph with $n$ vertices has at most $4 n-8$ edges and this upper bound is tight, which implies that the minimum vertex degree of any 1-planar graph is at most 7.

Let $H$ be a connected graph and $\mathcal{G}$ be a family of graphs. If for any graph $G \in \mathcal{G}, G$ contains a subgraph $K \simeq H$ such that

$$
\max _{x \in V(K)}\left\{d_{G}(x)\right\} \leq t_{h}<+\infty \text { and } \sum_{x \in V(K)} d_{G}(x) \leq t_{w}<+\infty,
$$

then we say that $H$ is light in $\mathcal{G}$, and otherwise say that $H$ is heavy in $\mathcal{G}$. The smallest integers $t_{h}$ and $t_{w}$ satisfying (1.1) are called the height and the weight of $H$ in the family $\mathcal{G}$, denoted by $h(H, \mathcal{G})$ and $w(H, \mathcal{G})$, respectively. By $\mathcal{L}(\mathcal{G})$, we denote the set of light graphs in the family $\mathcal{G}$. Throughout this paper, $\mathcal{P}_{\delta}^{1}(\varepsilon)\left(\right.$ resp. $\mathcal{P}_{\delta}(\varepsilon)$ ) denotes the family of 1-planar graphs (resp. planar graphs) with minimum vertex degree at least $\delta$ and minimum edge degree at least $\varepsilon$. If $\varepsilon=2 \delta$, we use the natation $\mathcal{P}_{\delta}^{1}$ (resp. $\mathcal{P}_{\delta}$ ) for short to represent $\mathcal{P}_{\delta}^{1}(\varepsilon)$ (resp. $\mathcal{P}_{\delta}(\varepsilon)$ ). Note that for the parameter $\mathcal{P}_{\delta}^{1}(\varepsilon)$, we need to assume that $\delta \leq 7$ and $\varepsilon \geq 2 \delta$.

The first complete description of the set of light graphs in the family of 1-planar graphs with high minimum degree was given in [9, 22]; there was proved that $\mathcal{L}\left(\mathcal{P}_{4}^{1}\right)=$ $\left\{P_{1}, P_{2}, P_{3}\right\}$. Fabrici and Madaras [9], Zhang, Liu and Wu [22], and Dong [8] together proved that $\mathcal{L}\left(\mathcal{P}_{5}^{1}\right)=\left\{P_{1}, P_{2}, P_{3}, P_{4}, S_{3}\right\}$, where $S_{3}$ is a 3-star. For the lightness of some graphs in the family $\mathcal{P}_{\delta}^{1}$ where $6 \leq \delta \leq 7$, the readers can refer to $[6,9,11,10,12,13,17$, 22, 26, 23].

In this paper, we investigate the lightness of some graphs in $\mathcal{P}_{\delta}^{1}(\varepsilon)$ with not only $\varepsilon=2 \delta$ but also $\varepsilon>2 \delta$, the later case of which has not been considered for the family of 1-planar graphs even before. Our motivation comes from the analogical results for planar graphs 
with minimum degree $\delta$ and minimum edge degree $2 \delta+1$ where $\delta \in\{3,4\}$. For example, Borodin proved in [5] that $\mathcal{L}\left(\mathcal{P}_{3}(7)\right)=\left\{P_{1}, P_{2}, P_{3}\right\}$ (also proved in [14] by Madaras and Škrekovski), and Mohar et al. [15] presented some light subgraphs in the class $\mathcal{P}_{4}(9)$.

In what follows, we show in Section 2.3 that $K_{4}^{-}$is light in the family $\mathcal{P}_{7}^{1}$ as well as in its superfamily $\mathcal{P}_{6}^{1}(13)$, and its height is at most 10 and at most 12 , respectively.

\section{The lightness of chordal 4-cycle}

\subsection{Basic terms}

In the following, we always assume that $G$ is a 1-planar graph that has been drawn on a plane so that every edge is crossed by at most one another edge and the number of crossings is as small as possible. The associated plane graph $G^{\times}$of $G$ is the plane graph that is obtained from $G$ by turning all crossings of $G$ into new 4 -vertices. A vertex in $G^{\times}$is false if it is not a vertex of $G$; otherwise, it is true. Similarly, by false (resp. true) face, we mean a face in $G^{\times}$that is incident with at least one false (resp. no false) vertices. Let $v$ and $f$ be a vertex and a face in $G^{\times}$. The function $\zeta(v)$ (resp. $\left.\zeta(f)\right)$ denotes the number of false vertices that are adjacent to $v$ (resp. incident with $f$ ) in $G^{\times}$.

For convenience, we introduce some specialized notations. Let $v$ be a false vertex in $G^{\times}$and let $v_{1}, v_{2}, v_{3}, v_{4}$ be its neighbors in a clockwise order. Define $f_{i}$ to be the face incident with $v v_{i}$ and $v v_{i+1}$, where subscripts are taken modulo 4. Note that if $d\left(f_{i}\right)=3$, then $v_{i} v_{i+1} \in E(G)$. In this case, let $f_{i}^{\prime}$ be the other face incident with the edge $v_{i} v_{i+1}$. If $d\left(f_{i}^{\prime}\right)=3$, then its third vertex will be denoted by $v_{i}^{\prime}$. Thus $v_{i}^{\prime}$ is a false vertex if and only if $f_{i}^{\prime}$ is false, in which case we denote a neighbor of $v_{i}$ (resp. $v_{i+1}$ ) in $G$ to be $v_{i}^{\prime \prime}$ (resp. $v_{i+1}^{\prime \prime}$ ), so that $v_{i} v_{i}^{\prime \prime}$ and $v_{i+1} v_{i+1}^{\prime \prime}$ are two edges in $G$ that crossed by each other at the point $v_{i}^{\prime}$. Denote the face that is incident with the path $v_{i} v_{i}^{\prime} v_{i+1}^{\prime \prime}\left(\right.$ resp. $\left.v_{i+1} v_{i}^{\prime} v_{i}^{\prime \prime}\right)$ in $G^{\times}$by $f_{i}^{L}\left(\operatorname{resp} . f_{i}^{R}\right)$.

While proving the lightness of a graph in a given family of graphs, usually, the discharging method is used. In the proof of this paper, based on this method we consider a hypothetical counterexample $G$ (a 1-planar graph) and then construct its associated plane graph $G^{\times}$. We first assign an initial charge $c$ to each element $x \in V\left(G^{\times}\right) \cup F\left(G^{\times}\right)$as follows:

$$
c(x)= \begin{cases}\alpha d_{G^{\times}}(x)-2(\alpha+\beta), & \text { if } x \in V\left(G^{\times}\right) ; \\ \beta d_{G^{\times}}(x)-2(\alpha+\beta), & \text { if } x \in F\left(G^{\times}\right),\end{cases}
$$

where $\alpha$ and $\beta$ are some prescribed positive numbers. By combining the Euler formula $\left|V\left(G^{\times}\right)\right|-\left|E\left(G^{\times}\right)\right|+\left|F\left(G^{\times}\right)\right|=2$ on $G^{\times}$and the relation $\sum_{v \in V\left(G^{\times}\right)} d_{G^{\times}}(v)=$ $\sum_{f \in F\left(G^{\times}\right)} d_{G^{\times}}(f)=2\left|E\left(G^{\times}\right)\right|$, we have $\sum_{x \in V\left(G^{\times}\right) \cup F\left(G^{\times}\right)} c(x)=-4(\alpha+\beta)<0$. We then redistribute the charge of the vertices and the faces of $G^{\times}$according to some discharging rules, which only move charge around but do not affect the total charges so that, after discharging, the final charge $c^{\prime}$ of each element in $V\left(G^{\times}\right) \cup F\left(G^{\times}\right)$is nonnegative. This leads to a contradiction that $\sum_{x \in V\left(G^{\times}\right) \cup F\left(G^{\times}\right)} c(x)=\sum_{x \in V\left(G^{\times}\right) \cup F\left(G^{\times}\right)} c^{\prime}(x) \geq$ 0 and completes the proof.

\subsection{A key discharging lemma}

Let $G$ be a 1-planar graph and let $v$ be a true vertex in its associated plane graph $G^{\times}$. Denote $F(v)$ to be the subgraph induced by the faces that are incident with $v$. Note that $F(v)$ can be decomposed into many parts, each of which is one of the five clusters in Figure 

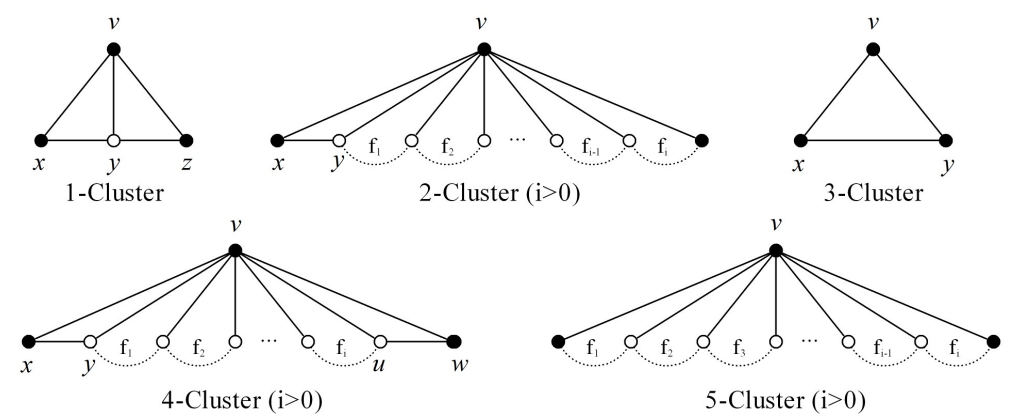

Figure 1: $F(v)$ can be decomposed into the combination of the above five clusters

1 , and any two parts of which are adjacent only if they have a common edge $v w$ such that $w$ is a true vertex. The hollow vertices in Figure 1 are false and the solid ones are true, and all the faces marked by $f_{i}$ are $4^{+}$-faces.

Lemma 2.1. Let $v$ be a $6^{+}$-vertex in the associated plane graph $G^{\times}$of a 1-planar graph G. Assign $v$ an initial charge $c(v)=\alpha d_{G^{\times}}(v)-2(\alpha+\beta)$, where $\alpha$ and $\beta$ are some prescribed positive numbers satisfying $2 \alpha \geq \beta$. Suppose that $v$ sends out charges only by the following three discharging rules:

Rule A $v$ transfers a charge of $\frac{2 \alpha-\beta}{3}$ to each incident 3-face in $G^{\times}$;

Rule B If $v$ is incident with two adjacent 3-faces $f_{1}=[x v z]$ and $f_{2}=[y v z]$ so that $z$ is a false vertex in $G^{\times}$, then $v$ sends the charge $\lambda$ to $z$;

Rule C If $v$ is incident with a 3-face $f_{1}=[x v z]$ sharing a common edge vz with a $4^{+}$-face $f_{2}$ so that $z$ is a false vertex in $G^{\times}$, then $v$ sends the charge $\mu$ to $z$.

Denote $c^{\prime}(v)$ to be the final charge of $v$ after applying the above rules. If $d_{G^{\times}}(v)$ is even with

$$
\begin{aligned}
& \lambda \leq\left(\frac{2}{3}-\frac{4}{d_{G^{\times}}(v)}\right)(\alpha+\beta), \\
& \mu=\frac{3}{4} \lambda,
\end{aligned}
$$

or $d_{G \times}(v) \geq 9$ is odd with

$$
\begin{aligned}
& \lambda \leq\left(\frac{2 d_{G^{\times}}(v)-12}{3 d_{G^{\times}}(v)-3}\right)(\alpha+\beta), \\
& \mu=\frac{1}{2} \lambda,
\end{aligned}
$$

or $d_{G \times}(v)=7$ with

$$
\lambda=\mu=\frac{\alpha+\beta}{12}
$$

then $c^{\prime}(v) \geq 0$. 
Proof. Denote $n_{i}$ to be the number of $i$-clusters contained in $F(v)$ and $m_{i}$ to be the charges sent out from $v$ through an $i$-cluster. By their definitions, one can easily observe that

$$
2 n_{1}+2 n_{2}+n_{3}+3 n_{4}+n_{5} \leq d_{G^{\times}}(v) .
$$

For the case when $d_{G^{\times}}(v)$ is even, by (2.7) and the choices of $\lambda, \mu$ as in (2.2) and (2.3), we have

$$
\begin{aligned}
c^{\prime}(v) & =c(v)-\frac{2 \alpha-\beta}{3} d_{G^{\times}}(v)-\sum_{i=1}^{5} n_{i} m_{i} \\
& =\frac{\alpha+\beta}{3} d_{G^{\times}}(v)-2(\alpha+\beta)-\lambda n_{1}-\mu n_{2}-2 \mu n_{4} \\
& \geq \frac{\alpha+\beta}{3} d_{G^{\times}}(v)-2(\alpha+\beta)-\frac{\lambda}{2}\left(2 n_{1}+2 n_{2}+n_{3}+3 n_{4}+n_{5}\right) \\
& \geq \frac{\alpha+\beta}{3} d_{G^{\times}}(v)-2(\alpha+\beta)-\frac{\lambda}{2} d_{G^{\times}}(v) \\
& \geq \frac{\alpha+\beta}{3} d_{G^{\times}}(v)-2(\alpha+\beta)-\left(\frac{1}{3}-\frac{2}{d_{G^{\times}}(v)}\right)(\alpha+\beta) d_{G^{\times}}(v) \\
& =0 .
\end{aligned}
$$

Now, we consider the case when $d_{G^{\times}}(v)$ is odd. Here, note that

$$
n_{2}+n_{3}+n_{4}+n_{5} \geq 1
$$

since any copy of a 1-cluster consists even number of faces incident with $v$. By (2.7), (2.8) and the choices of $\lambda, \mu$ as in (2.4) and (2.5), we have

$$
\begin{aligned}
& c^{\prime}(v)=c(v)-\frac{2 \alpha-\beta}{3} d_{G^{\times}}(v)-\sum_{i=1}^{5} n_{i} m_{i} \\
& =\frac{\alpha+\beta}{3} d_{G^{\times}}(v)-2(\alpha+\beta)-\lambda n_{1}-\mu n_{2}-2 \mu n_{4} \\
& =\frac{\alpha+\beta}{3} d_{G \times}(v)-2(\alpha+\beta)-\frac{\lambda}{2}\left(2 n_{1}+2 n_{2}+n_{3}+3 n_{4}+n_{5}\right)+ \\
& \frac{\lambda}{2}\left(n_{2}+n_{3}+n_{4}+n_{5}\right) \\
& \geq \frac{\alpha+\beta}{3} d_{G \times}(v)-2(\alpha+\beta)-\frac{\lambda}{2} d_{G \times}(v)+\frac{\lambda}{2} \\
& \geq \frac{\alpha+\beta}{3} d_{G^{\times}}(v)-2(\alpha+\beta)-\left(\frac{d_{G^{\times}}(v)-6}{3 d_{G^{\times}}(v)-3}\right)(\alpha+\beta)\left(d_{G^{\times}}(v)-1\right) \\
& =0 \text {. }
\end{aligned}
$$

For the particular case when $d_{G^{\times}}(v)=7$, we can deduce from (2.7) that

$$
n_{1}+n_{2}+2 n_{4}=\left\lfloor\frac{2 n_{1}+2 n_{2}+3 n_{4}}{2}\right\rfloor+\left\lceil\frac{1}{2} n_{4}\right\rceil \leq\left\lfloor\frac{7}{2}\right\rfloor+\left\lceil\frac{1}{2}\left\lfloor\frac{7}{3}\right\rfloor\right\rceil=4 .
$$


Thus by (2.9) along with the choices of $\lambda$ and $\mu$ as in the equation (2.6), we have

$$
\begin{aligned}
c^{\prime}(v) & =c(v)-\frac{2 \alpha-\beta}{3} d_{G \times}(v)-\sum_{i=1}^{5} n_{i} m_{i} \\
& =\frac{\alpha+\beta}{3}-\lambda n_{1}-\mu n_{2}-2 \mu n_{4} \\
& =\frac{\alpha+\beta}{3}-\lambda\left(n_{1}+n_{2}+2 n_{4}\right) \\
& \geq \frac{\alpha+\beta}{3}-4 \lambda \\
& =0 .
\end{aligned}
$$

Consequently, we complete the proof of this lemma.

\subsection{The height of chordal 4-cycle in $\mathcal{P}_{6}^{1}(13)$ and $\mathcal{P}_{7}^{1}$}

Theorem 2.2. Each 1-planar graph with minimum degree at least 6 contains at least one of the following configurations:

(a) a pair of adjacent vertices of degree 6;

(b) a 4-cycle $C=\left[x_{1} x_{2} x_{3} x_{4}\right]$ of the type $(6, \leq 12, \leq 8, \leq 12)$ with a chord $x_{1} x_{3}$;

(c) a 4-cycle $C=\left[x_{1} x_{2} x_{3} x_{4}\right]$ of the type $(7, \leq 10, \leq 8, \leq 10)$ with a chord $x_{1} x_{3}$.

Proof. The proof of the theorem is carried out by the discharging method as described in Section 2.1. Suppose $G$ is a counterexample to the theorem. Consider the associated plane graph $G^{\times}$of $G$. Assign the charges to each element $x \in V\left(G^{\times}\right) \cup F\left(G^{\times}\right)$as mentioned in the inequation (2.1) of Section 2.1 by choosing $\alpha=2$ and $\beta=3$. If $v$ is a true vertex in $G$, then $d_{G^{\times}}(v)=d_{G}(v)$, so in the following we use $d(v)$ for short to represent both of the two notions. A big vertex, semi-big vertex, intermediate vertex and semi-intermediate vertex refer to a vertex $v \in V\left(G^{\times}\right)$with $d(v) \geq 13, d(v) \geq 11,6 \leq d(v) \leq 12$ and $6 \leq d(v) \leq 10$, respectively. Therefore, a true vertex in $G^{\times}$is either big or intermediate, and an intermediate vertex in $G^{\times}$is either semi-big or semi-intermediate. By big face, we denote a face $f \in F\left(G^{\times}\right)$with degree at least 4 . Now, we define the discharging rules as follows.

Rule 1 Each $6^{+}$-vertex sends $\frac{1}{3}$ to each incident face;

Rule 2 Each 4-vertex sends $\frac{1}{3}$ to each incident false 3-face;

Rule 3 Each big face sends $\frac{11}{12}$ to each incident 4-vertex;

Rule 4 Let $f$ be a big face having a common edge $x y$ with a false 3-face $g=[x y z]$. If $z$ is a 4-vertex, then $f$ sends $\frac{5}{12}$ to $z$ through $x y$;

Rule 5 Let $f$ be a big face having a common edge $x y$ with a false 3 -face $g=[x y z]$. If $x$ is a 4-vertex and $y z$ is incident with another false 3-face $h=[y z u]$, then $f$ sends $\frac{5}{24}$ to $u$ through $x y$ and $y z$;

Rule 6 Let $f=[x y z]$ be a true 3-face having a common edge $y z$ with a false 3-face $g=[u y z]$. If $d(x) \geq 11$, then $x$ sends $\frac{5}{12}$ to $u$ through $y z$; 
Rule 7 Let $f=[x y z]$ and $g=[u y z]$ be two adjacent false 3-faces, and let $z$ be a 4-vertex. Suppose $y u$ is incident with another false 3-face $h=[y u w]$ so that $y y^{\prime}$ crosses $u u^{\prime}$ in $G$ at $w$. If at least one of the following four occasions appears in $G^{\times}$

- $d(x) \geq 13, \min \{d(u), d(y)\}=6, \max \{d(u), d(y)\} \leq 8$ and $y, u, u^{\prime}$ are all intermediate vertices with $y u^{\prime} \in E\left(G^{\times}\right)$;

- $d(x) \geq 13, \min \{d(u), d(y)\}=6, \max \{d(u), d(y)\} \leq 8$ and $y, u, y^{\prime}$ are all intermediate vertices with $u y^{\prime} \in E\left(G^{\times}\right)$;

- $d(x) \geq 11, \min \{d(u), d(y)\}=7, \max \{d(u), d(y)\} \leq 8$ and $y, u, u^{\prime}$ are all semi-intermediate vertices with $y u^{\prime} \in E\left(G^{\times}\right)$;

- $d(x) \geq 11, \min \{d(u), d(y)\}=7, \max \{d(u), d(y)\} \leq 8$ and $y, u, y^{\prime}$ are all semi-intermediate vertices with $u y^{\prime} \in E\left(G^{\times}\right)$,

then $x$ sends $\frac{5}{24}$ to $w$ through $y z$ and $y u$;

Rule 8 Let $f=[x y z]$ and $g=[u y z]$ be two adjacent false 3-faces. If $z$ is a 4-vertex, then $y$ sends to $z$ a charge of

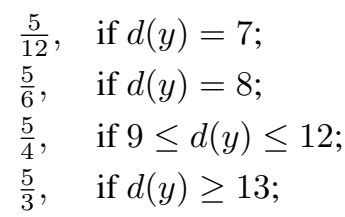

Rule 9 Let $f=[x y z]$ be a false 3-face having a common edge $y z$ with a big face $\beta$. If $z$ is a 4-vertex, then $y$ sends to $z$ a charge of

$$
\begin{array}{ll}
\frac{5}{12}, & \text { if } d(y)=7 ; \\
\frac{5}{8}, & \text { if } 8 \leq d(y) \leq 12 ; \\
\frac{5}{6}, & \text { if } d(y) \geq 13 .
\end{array}
$$

In the following, we estimate the final charge $c^{\prime}$ of vertices and faces after the charge redistribution and prove $c^{\prime}(x) \geq 0$ for each $x \in V\left(G^{\times}\right) \cup F\left(G^{\times}\right)$. By Rules 1 and 2, any 3 -face $f$ in $G^{\times}$receive $\frac{1}{3}$ from each of its incident vertices, which implies the final charge of $f$ is exactly zero. For a big face $f$ in $G^{\times}$(recall that $\zeta(f)$ denotes the number of 4-vertices incident with $f$ ), it would send $\frac{11}{12} \zeta(f)$ to its incident 4-vertices by Rule 3 . Besides, if $f$ is incident with a 4-vertex $v$, then $f$ send out $2 \times \frac{5}{24}=\frac{5}{12}$ through $u v$ and $v w$ by Rule 5, where $u$ and $w$ denote the neighbors of $v$ on the boundary of $f$. Since $f$ is incident with $d(f)-2 \zeta(f)$ true edges (namely, an edge of $G^{\times}$containing no 4-vertex), by Rule 4, a total charge of $\frac{5}{12}(d(f)-2 \zeta(f))$ would be sent out from $f$ through the true edges incident with $f$. On the other hand, $f$ receive $\frac{1}{3}$ from each of $d(f)-\zeta(f)$ true vertices incident with it. Since $\zeta(f) \leq \frac{d(f)}{2}, c^{\prime}(f) \geq 3 d(f)-10-\frac{11}{12} \zeta(f)-\frac{5}{12} \zeta(f)-\frac{5}{12}(d(f)-2 \zeta(f))+\frac{1}{3}(d(f)-\zeta(f))=$ $\frac{35}{12} d(f)-\frac{5}{6} \zeta(f)-10 \geq \frac{5}{2} d(f)-10 \geq 0$ for $d(f) \geq 4$.

By Lemma 2.1 along with Rules 1,8 and 9, one can check that $c^{\prime}(v) \geq 0$ for all vertices of degree between 6 and 10. For a big vertex $v$ in $G^{\times}$, denote $F(v)$ to be the subgraph induced by the faces that are incident with $v$. As we state at the beginning of Section 2.2, $F(v)$ can be decomposed into a combination of the five clusters in Figure 1. By $n_{i}$ and $m_{i}$, we denote the number of $i$-clusters contained in $F(v)$ and the charges sent out from $v$ through an $i$-cluster. If there is a 2-cluster in $F(v)$, then $v$ send $\frac{5}{6}$ to $y$ (see Figure 1) by Rule 9 and at most $\frac{5}{24}$ through $x y$ by Rule 7 , so $m_{2} \leq \frac{5}{6}+\frac{5}{24}=\frac{25}{24}$. Similarly, we can prove 
that $m_{3} \leq \frac{5}{12}$ by Rule $6, m_{4} \leq 2 \times \frac{5}{6}+2 \times \frac{5}{24}=\frac{25}{12}$ by Rules 7,9 and $m_{5}=0$. We now estimate the value of $m_{1}$ much more carefully. First, we show the following observation.

Observation. If $v$ is incident with a 1-cluster as in Figure 1 and has sent out some charge through $x y$ by Rule 7 , then it would not send out any charge through the edge yz.

Proof. Denote $u$ to be the neighbor of $v$ in $G$ such that $u v$ crosses $x z$ in $G$ at the point $y$. Suppose, on the contrary, that $v$ send out some charge through $y z$. By the definitions of the rules, $u y z$ is a 3 -face in $G^{\times}$and $z$ is an intermediate vertex in $G^{\times}$. Since $v$ has sent out charge through $x y$ by Rule 7, by the definition of Rule 7, we have $x u \in E\left(G^{\times}\right)$, $\min \{d(x), d(u)\}=6$ and $\max \{d(x), d(u)\} \leq 8$. Furthermore, $x u$ is also incident with a 3-cycle, say $x u w$, in $G$ such that $w$ is an intermediate vertex in $G^{\times}$different from $z$. Now, the four distinct vertices $x, z, u, w$ form a 4-cycle $[u w x z]$ in $G$ with a chord $u x$, and therefore, the configuration (b) occurs in $G$. This contradiction verifies this observation.

By Rules 7, 8 and the above observation, we immediately have $m_{1} \leq \frac{5}{3}+\frac{5}{24}=\frac{15}{8}$. Therefore, by Rule 1 and the inequality (2.7) in Section 2.1, we have $c^{\prime}(v) \geq 2 d(v)-$ $10-\frac{1}{3} d(v)-\frac{15}{8} n_{1}-\frac{25}{24} n_{2}-\frac{5}{12} n_{3}-\frac{25}{12} n_{4} \geq \frac{5}{3} d(v)-10-\frac{15}{16}\left(2 n_{1}+2 n_{2}+n_{3}+\right.$ $\left.3 n_{4}+n_{5}\right)+\frac{25}{48}\left(n_{2}+n_{3}+n_{4}+n_{5}\right) \geq \frac{35}{48} d(v)-10+\frac{25}{48}\left(n_{2}+n_{3}+n_{4}+n_{5}\right)>0$ for $d(v) \geq 14$. If $d(v)=13$, then by the inequality (2.8) in Section 2.1, we also have $c^{\prime}(v) \geq \frac{35}{48} d(v)-10+\frac{25}{48}=0$ in final. For vertices of degree 11 or 12 (they are semi-big but not big), we can also check the nonnegativity of their final charges. Proof of them are left to the readers, since they use the same argument as in the previous analysis on the big vertices.

Now, the only missed case is when $v$ is a 4-vertex in $G^{\times}$(namely, $v$ is a false vertex). As we know, the initial charge of a 4-vertex $v$ is -2 , so if $v$ is incident with at least three big faces, then by Rules 2 and 3, the final charge $c^{\prime}(v)$ of $v$ is at least $-2-\frac{1}{3}+3 \times \frac{11}{12}=\frac{5}{12}>0$. In the following, we discuss three other cases.

Case 1. $v$ is incident with exactly two 3-faces.

First, suppose that $f_{1}$ and $f_{2}$ are 3 -faces. Since no two 6-vertices are adjacent in $G$, at least two of $v_{1}, v_{2}$ and $v_{3}$ are $7^{+}$-vertices. Thus by Rules 8 and 9 , each of the two $7^{+}$vertices among $v_{1}, v_{2}$ and $v_{3}$ would send at least $\frac{5}{12}$ to $v$. Therefore, $c^{\prime}(v) \geq-2-2 \times \frac{1}{3}+$ $2 \times \frac{11}{12}+2 \times \frac{5}{12}=0$ by Rules $2,3,8$ and 9 .

Second, suppose that $f_{1}$ and $f_{3}$ are 3 -faces. In this case, one can also show that there are at least two $7^{+}$-vertices among $v_{1}, v_{2}, v_{3}$ and $v_{4}$. Thus by Rules 2, 3,8 and 9, we still have $c^{\prime}(v) \geq-2-2 \times \frac{1}{3}+2 \times \frac{11}{12}+2 \times \frac{5}{12}=0$.

Case 2. $v$ is incident with exactly three 3-faces.

Without loss of generality, we assume that $f_{1}, f_{2}, f_{3}$ are 3 -faces and $f_{4}$ is a $4^{+}$-face (recall the definitions of $f_{i}$ in Section 2.1). By Rule 3, $f_{4}$ shall send $\frac{11}{12}$ to $v$.

First, suppose that at least two of $v_{1}, v_{2}, v_{3}$ and $v_{4}$, say $v_{1}$ and $v_{4}$ (other cases can be dealt with similarly), are big vertices. Thus at least one of $v_{2}$ and $v_{3}$ must be $7^{+}$-vertex since they are adjacent in $G$. Therefore, by Rules 2,3 and $9, c^{\prime}(v) \geq-2-3 \times \frac{1}{3}+2 \times \frac{5}{6}+\frac{5}{12}+\frac{11}{12}=$ 0 .

Next, suppose that only one of $v_{1}, v_{2}, v_{3}$ and $v_{4}$ is big vertex. If $v_{2}$ or $v_{3}$, say $v_{2}$, is big, then at least one of $v_{1}, v_{3}$ and $v_{4}$ should be a $7^{+}$-vertex since they three form a 3-path in $G$. By Rules 2, 3, 8 and 9, $c^{\prime}(v) \geq-2-3 \times \frac{1}{3}+\frac{5}{3}+\frac{5}{12}+\frac{11}{12}=0$. If $v_{1}$ or $v_{4}$, say $v_{1}$, is big, then all of $v_{2}, v_{3}$ and $v_{4}$ are intermediate. If they are all $7^{+}$-vertices, then $c^{\prime}(v) \geq-2-3 \times \frac{1}{3}+\frac{5}{6}+3 \times \frac{5}{12}+\frac{11}{12}=0$ by Rules 2,8 and 9 . Thus we assume that at 
least one of $v_{2}, v_{3}$ and $v_{4}$ is a 6-vertex. Here, we only consider the case when $d\left(v_{3}\right)=6$ and leave the discussions on the rest two cases to the readers, since they are quite similar. First, suppose $d\left(v_{2}\right) \geq 9$. By Rules 3 and $8, v_{1}, v_{2}$ and $f_{4}$ shall send $\frac{5}{6}, \frac{5}{4}$ and $\frac{11}{12}$ to $v$, respectively, thus, $c^{\prime}(v) \geq-2-3 \times \frac{1}{3}+\frac{5}{6}+\frac{5}{4}+\frac{11}{12}=0$. Second, suppose $d\left(v_{2}\right) \leq 8$. We now consider the face $f_{2}^{\prime}$ (recall its definition in Section 2.1). If $f_{2}^{\prime}$ is a big face in $G^{\times}$, then by Rule $4, f_{2}^{\prime}$ sends $\frac{5}{12}$ to $v$ through the edge $v_{2} v_{3}$. If $f_{2}^{\prime}$ is a true 3 -face, then $v_{2}^{\prime}$ (recall the corresponding definition in Section 2.1) must be a big vertex, because otherwise the configuration (b) would appear in $G$, meanwhile, $v$ receives $\frac{5}{12}$ from $v_{2}^{\prime}$ through the edge $v_{2} v_{3}$ by Rule 6. If $f_{2}^{\prime}$ is false 3 -face, then we consider the faces $f_{2}^{L}$ and $f_{2}^{R}$ (recall their definitions in Section 2.1). If $f_{2}^{L}$ is a big face, then by Rule 5, $f_{2}^{L}$ sends $\frac{5}{24}$ to $v$ through the edge $v_{2} v_{3}$. If $f_{2}^{L}$ is a 3 -face, then it must be false since it is incident with a false vertex $v_{2}^{\prime}$. Since $v_{2}, v_{3}, v_{4}$ and $v_{3}^{\prime \prime}$ (recall the definitions of $v_{i}^{\prime \prime}$ in Section 2.1) form a chordal 4-cycle with a chord $v_{2} v_{3}$ in $G, v_{3}^{\prime \prime}$ must be a big vertex, and then $v$ shall receive $\frac{5}{24}$ from $v_{3}^{\prime \prime}$ through the edge $v_{2} v_{3}$ by Rule 7. Similarly, $v$ would receive another $\frac{5}{24}$ through the edge $v_{2} v_{3}$ from either the face $f_{2}^{R}$ or the vertex $v_{2}^{\prime \prime}$. Hence, through the edge $v_{2} v_{3}, v$ shall totally receive a charge of $2 \times \frac{5}{24}=\frac{5}{12}$. Since neither $v_{2}$ nor $v_{4}$ can be a 6-vertex (because each of them is adjacent to the 6-vertex $v_{3}$ in $G$ ), each of $v_{2}$ and $v_{4}$ shall send $\frac{5}{12}$ to $v$ by Rules 8 and 9 . Thus, by Rules 2, 3 and 9, we still have $c^{\prime}(v) \geq-2-3 \times \frac{1}{3}+\frac{5}{12}+2 \times \frac{5}{12}+\frac{5}{6}+\frac{11}{12}=0$.

At last, suppose none of $v_{1}, v_{2}, v_{3}$ and $v_{4}$ is big. If $v_{2}$ or $v_{3}$, say $v_{2}$, is a 6-vertex, then $v_{1}, v_{3}$ and $v_{4}$ are $7^{+}$-vertices since each of them is adjacent to $v_{2}$. Furthermore, $d\left(v_{3}\right) \geq 9$, because otherwise the configuration (b) would appear in $G$, so by Rules 2, 3, 8 and 9, we have $c^{\prime}(v) \geq-2-3 \times \frac{1}{3}+2 \times \frac{5}{12}+\frac{5}{4}+\frac{11}{12}=0$. We now assume $\min \left\{d\left(v_{2}\right), d\left(v_{3}\right)\right\} \geq 7$. If $\max \left\{d\left(v_{2}\right), d\left(v_{3}\right)\right\} \geq 9$ (without loss of generality, assume $d\left(v_{3}\right) \geq 9$ ), then by Rules 2,3 , 8 and $9, c^{\prime}(v) \geq-2-3 \times \frac{1}{3}+2 \times \frac{5}{4}+\frac{11}{12}>0$ when $d\left(v_{2}\right) \geq 9, c^{\prime}(v) \geq-2-3 \times \frac{1}{3}+2 \times \frac{5}{12}+$ $\frac{5}{4}+\frac{11}{12}=0$ when $d\left(v_{2}\right) \leq 8$ and $d\left(v_{1}\right) \geq 7$, and $c^{\prime}(v) \geq-2-3 \times \frac{1}{3}+\frac{5}{12}+\frac{5}{12}+\frac{5}{4}+\frac{11}{12}=0$ when $d\left(v_{2}\right) \leq 8$ and $d\left(v_{1}\right)=6$ (note that in this case, a charge of at least $\frac{5}{12}$ shall be transferred to $v$ through the edge $\left.v_{1} v_{2}\right)$. Therefore, we assume $\max \left\{d\left(v_{2}\right), d\left(v_{3}\right)\right\} \leq 8$ in the following. First, suppose at least one of $v_{2}$ and $v_{3}$ is a 8-vertex. Without loss of generality, suppose $d\left(v_{2}\right)=7$ and $d\left(v_{3}\right)=8$. By Rule $8, v_{2}$ and $v_{3}$ shall send $\frac{5}{12}$ and $\frac{5}{6}$ to $v$, respectively. By a similar argument as above, $v$ shall also receive $\frac{5}{12}$ either from the vertex $v_{1}$ when $d\left(v_{1}\right) \geq 7$ or through the edge $v_{1} v_{2}$ when $d\left(v_{(1}\right)=6$, and another $\frac{5}{12}$ either from the vertex $v_{4}$ when $d\left(v_{4}\right) \geq 7$ or through the edge $v_{3} v_{4}$ when $d\left(v_{(4}\right)=6$. Thus, by Rules 2 and 3, we have $c^{\prime}(v) \geq-2-3 \times \frac{1}{3}+\frac{5}{12}+\frac{5}{6}+2 \times \frac{5}{12}+\frac{11}{12}=0$.

Second, suppose $d\left(v_{2}\right)=d\left(v_{3}\right)=7$. Under this hypothesis, at least one of $v_{1}$ and $v_{4}$ should be semi-big, because otherwise a configuration (c) would appear in $G$. If $v_{1}$ and $v_{4}$ are $8^{+}$-vertices, then by Rules $2,3,8$ and $9, c^{\prime}(v) \geq-2-3 \times \frac{1}{3}+2 \times \frac{5}{12}+2 \times \frac{5}{8}+\frac{11}{12}=0$. If one of $v_{1}$ and $v_{4}$, say $v_{4}$, is a $7^{-}$-vertex, then by a similar argument as before, we can show that $v$ receives $\frac{5}{12}$ through the edges $v_{1} v_{2}$ and another $\frac{5}{12}$ through the edges $v_{2} v_{3}$. Therefore, by Rules $2,3,8$ and 9, we have $c^{\prime}(v) \geq-2-3 \times \frac{1}{3}+2 \times \frac{5}{12}+\frac{5}{8}+2 \times \frac{5}{12}+\frac{11}{12}>0$.

Case 3. $v$ is incident with four 3-faces.

If at least two of $v_{1}, v_{2}, v_{3}$ and $v_{4}$ are big vertices, then by Rules 2 and $8, c^{\prime}(v) \geq$ $-2-4 \times \frac{1}{3}+2 \times \frac{5}{3}=0$.

If only one of $v_{1}, v_{2}, v_{3}, v_{4}$, say $v_{1}$, is a big vertex, then we can assume that $v_{2}, v_{3}$ and $v_{4}$ are $7^{+}$-vertices. Otherwise, without loss of generality, suppose $d\left(v_{2}\right)=6$. Since no two 6 -vertices are adjacent in $G, d\left(v_{3}\right) \geq 7$ and $d\left(v_{4}\right) \geq 7$. If $v_{3}$ and $v_{4}$ are $8^{+}$-vertices, then $c^{\prime}(v) \geq-2-4 \times \frac{1}{3}+\frac{5}{3}+2 \times \frac{5}{6}=0$ by Rules 2 and 8 . We now assume that one of $v_{3}$ and $v_{4}$, say $v_{3}$, is a 7-vertex. If now $d\left(v_{4}\right) \geq 9$, then $c^{\prime}(v) \geq-2-4 \times \frac{1}{3}+\frac{5}{3}+\frac{5}{12}+\frac{5}{4}=0$ 
Rules 2 and 8 . If $d\left(v_{4}\right) \leq 8$, then by a similar argument as in Case 1 , we can show that $v$ receives $\frac{5}{12}$ through the edges $v_{2} v_{3}$ and another $\frac{5}{12}$ through the edges $v_{3} v_{4}$. Therefore, by Rules 2 and $8, c^{\prime}(v) \geq-2-4 \times \frac{1}{3}+\frac{5}{3}+2 \times \frac{5}{12}+2 \times \frac{5}{12}=0$. Hence, we can assume $\min \left\{d\left(v_{2}\right), d\left(v_{3}\right), d\left(v_{4}\right)\right\} \geq 7$. If at least one of $v_{2}, v_{3}$ and $v_{4}$ is a $8^{+}$-vertex, then by Rules 2 and $8, c^{\prime}(v) \geq-2-4 \times \frac{1}{3}+2 \times \frac{5}{12}+\frac{5}{6}=0$. If $d\left(v_{2}\right)=d\left(v_{3}\right)=d\left(v_{4}\right)=7$, then by a similar argument as in Case 1 , one can show that a charge of $\frac{5}{12}$ would be transferred to $v$ through each of the edges $v_{2} v_{3}$ and $v_{3} v_{4}$. Hence, by Rules 2 and 8 , we have $c^{\prime}(v) \geq$ $-2-4 \times \frac{1}{3}+3 \times \frac{5}{12}+2 \times \frac{5}{12}>0$.

We now consider the last case when $v_{1}, v_{2}, v_{3}$ and $v_{4}$ are intermediate vertices. If they all are $8^{+}$-vertices, then by Rules 2 and $8, c^{\prime}(v) \geq-2-4 \times \frac{1}{3}+4 \times \frac{5}{6}=0$. If one of them, say $v_{1}$, is a 6-vertex, then $v_{2}, v_{3}$ and $v_{4}$ are $9^{+}$-vertex, because otherwise the configuration (b) would appear in $G$. This implies that $c^{\prime}(v) \geq-2-4 \times \frac{1}{3}+3 \times \frac{5}{4}>0$ by Rules 2 and 8 . We now assume that $d\left(v_{1}\right)=7$ and $\min \left\{d\left(v_{2}\right), d\left(v_{3}\right), d\left(v_{4}\right)\right\} \geq 7$. If at least two of $v_{2}, v_{3}$ and $v_{4}$ are $9^{+}$-vertices, then by Rules 2 and $8, c^{\prime}(v) \geq-2-4 \times \frac{1}{3}+2 \times \frac{5}{12}+2 \times \frac{5}{4}=0$. Thus, we assume that at least two of $v_{2}, v_{3}, v_{4}$, say $v_{2}$ and $v_{3}$, are $8^{-}$-vertices. In this case, $v_{4}$ should be a semi-big vertex because otherwise the configuration (c) would occur in $G$. If $d\left(v_{2}\right)=d\left(v_{3}\right)=8$, then by Rules 2 and $8, c^{\prime}(v) \geq-2-4 \times \frac{1}{3}+\frac{5}{12}+2 \times \frac{5}{6}+\frac{5}{4}=0$. If $\min \left\{d\left(v_{2}\right), d\left(v_{3}\right)\right\}=7$, then by a similar argument as in Case 1 , one can prove that a charge of $\frac{5}{12}$ would be transferred to $v$ through each of the edges $v_{1} v_{2}$ and $v_{2} v_{3}$. This implies that $c^{\prime}(v) \geq-2-4 \times \frac{1}{3}+3 \times \frac{5}{12}+2 \times \frac{5}{12}+\frac{5}{4}=0$.

Hence, we deduce that $\sum_{x \in V\left(G^{\times}\right) \cup F\left(G^{\times}\right)} c^{\prime}(x) \geq 0$. This contradiction completes the proof.

Corollary 2.3. $K_{4}^{-} \in \mathcal{L}\left(\mathcal{P}_{6}^{1}(13)\right)$ and $h\left(K_{4}^{-}, \mathcal{P}_{6}^{1}(13)\right) \leq 12$.

Corollary 2.4. $K_{4}^{-} \in \mathcal{L}\left(\mathcal{P}_{7}^{1}\right)$ and $h\left(K_{4}^{-}, \mathcal{P}_{7}^{1}\right) \leq 10$.

Corollary 2.5. $h\left(P_{2}, P_{6}^{1}\right) \leq 8$ and $w\left(P_{2}, P_{6}^{1}\right) \leq 15$.

\section{References}

[1] M. O. Albertson and B. Mohar, Coloring vertices and faces of locally planar graphs. Graph. Combinator. 22 (2006), 289-295.

[2] J. A. Bondy and U. S. R. Murty, Graph Theory with Applications, North-Holland, New York, 1976.

[3] O. V. Borodin, Solution of Ringel's problems on the vertex-face coloring of plane graphs and on the coloring of 1-planar graphs (in Russian), Diskret. Analiz 41 (1984), 12-26.

[4] O. V. Borodin, A New Proof of the six Color Theorem, J. Graph Theory 19 (1995), 507-521.

[5] O.V. Borodin, Minimal vertex degree sum of a 3-path in plane maps, Discuss. Math. Graph Theory 17 (1997), 279-284.

[6] O. V. Borodin, I. G. Dmitriev and A. O. Ivanova, The height of a 4-cycle in triangle-free 1planar graphs with minimum degree 5, J. Appl. Industrial Math. 3 (2009), 28-31.

[7] O. V. Borodin, A. V. Kostochka, A. Raspaud and E. Sopena, Acyclic colouring of 1-planar graphs, Discrete Appl. Mat. 114 (2001), 29-41.

[8] W. Dong, Light paths of 1-planar graphs with bounded minimum degree, manuscript.

[9] I. Fabrici and T. Madaras, The structure of 1-planar graphs, Discrete Math. 307 (2007), 854865 . 
[10] D. Hudák and T. Madaras, On local structures of 1-planar graphs of minimum degree 5 and girth 4, Discuss. Math. Graph Theory 29 (2009), 385-400.

[11] D. Hudák and T. Madaras, On local properties of 1-planar graphs with high minimum degree, Ars Math. Contemp. 4 (2011), 245-254.

[12] V. P. Korzhik, Minimal non-1-planar graphs, Discrete Math. 308 (2008), 1319-1327.

[13] V. P. Korzhik and B. Mohar, Minimal obstructions for 1-immersions and hardness of 1-planarity test, Springer Lect. Notes Comput. Sci. 5417 (2009), 302-312, 2009.

[14] T. Madaras and R. Škrekovski, Heavy paths, light stars and big melons, Discrete Math. 286 (2004), 115-131.

[15] B. Mohar, R. Škrekovski and H.-J. Voss, Light subgraphs in planar graphs of minimum degree 4 and edge-degree 9, J. Graph Theory 44 (2003), 261-295.

[16] G. Ringel, Ein sechsfarbenproblem auf der Kugel (in German), Abh. Math. Sem. Hamburg. Univ. 29 (1965), 107-117.

[17] Y. Suzuki, Optimal 1-planar graphs which triangulate other surfaces, Discrete Math. 310 (2010), 6-11.

[18] W. Wang and K.-W. Lih, Coupled choosability of plane graphs, J. Graph Theory 58 (2008), 27-44.

[19] X. Zhang and G. Liu, On edge colorings of 1-planar graphs without chordal 5-cycles, Ars Combin. 104 (2012), 431-436.

[20] X. Zhang and G. Liu, On edge colorings of 1-planar graphs without adjacent triangles, Inform. Process. Lett. 112 (2012), 138-142.

[21] X. Zhang, G. Liu and J.-L. Wu, Edge coloring of triangle-free 1-planar graphs (in Chinese), Journal of Shandong University (Natural Science) 45 (2010), 15-17.

[22] X. Zhang, G. Liu and J.-L. Wu, Structural properties of 1-planar graphs and an application to acyclic edge coloring (in Chinese), Scientia Sinica Mathematica 40 (2010), 1025-1032.

[23] X. Zhang, G. Liu and J.-L. Wu, Light subgraphs in the family of 1-planar graphs with high minimum degree, Acta Math. Sinica, English Series 28 (2012), 1155-1168.

[24] X. Zhang, G. Liu and J.-L. Wu, On the linear arboricity of 1-planar graphs, OR Transactions 15 (2011), 38-44.

[25] X. Zhang and J.-L. Wu, Edge coloring of 1-planar graphs, Inform. Process. Lett. 111 (2011), 124-128.

[26] X. Zhang X, J.-L. Wu and G. Liu, New upper bounds for the heights of some light subgraphs in 1-planar graphs with high minimum degree, Discrete Math. Theoret. Comp. Sc. 13 (2011), $9-16$.

[27] X. Zhang, J.-L. Wu, G. Liu, List edge and list total coloring of 1-planar graphs, Front. Math. China 7 (2012), 1005-1018.

[28] X. Zhang, Y. Yu and G. Liu, On ( $p, 1)$-total labelling of 1-planar graphs, Cent. Eur. J. Math. 9 (2011), 1424-1434. 